\title{
RIGIDEZ PÓS-TRAUMÁTICA DO COTOVELO
}

\author{
POST-TRAUMATIC STIFFNESS OF THE ELBOW
}

\section{RESUMO}

Rigidez do cotovelo é uma complicação comum, após trauma articular causando comprometimento da função do membro superior. A gravidade da disfunção depende da natureza do trauma inicial e da terapêutica empregada. A avaliação clínica e através de exames complementares adequados são fundamentais no planejamento terapêutico. Diversas modalidades cirúrgicas são disponíveis atualmente e a indicação deve ser individualizada de acordo com o perfil do paciente, do grau de limitação articular e habilidade do cirurgião. Incongruência e degeneração articular influenciam negativamente o prognóstico, porém, a presença de ossificação heterotópica isolada relaciona-se a um prognóstico cirúrgico favorável.

Descritores - Cotovelo/lesões; Cotovelo/cirurgia. Ferimentos e lesões

\section{INTRODUÇÃO}

Rigidez do cotovelo é uma afecção comum, após traumatismo articular podendo causar comprometimento substancial da função do membro superior ${ }^{(1)}$. Diversas situações clínicas podem proporcionar perda de movimento no cotovelo. O sucesso de seu tratamento depende de uma avaliação clínica adequada que determine as alterações anatômicas envolvidas no processo patológico, permitindo ao cirurgião intervir adequadamente ${ }^{(2)}$.

\section{Etiologia}

A propensão da articulação do cotovelo a desenvolver rigidez pós-trauma é reconhecida pelos ortopedistas, mesmo após traumas leves. As principais razões que expõe a articulação do cotovelo a esta complicação

\section{ABSTRACT}

Stiff elbow is a common problem after articular trauma, causing functional impairment of the upper limb. The gravity of the dysfunction depends on the nature of the initial trauma and the treatment employed. Rigorous clinical evaluation and complementary exams are essential to creating a therapeutic plan. Several surgical techniques are available and a recommendation must be made according to patient characteristics, degree of joint dysfunction and the surgeon's skill. Articular incongruence and degeneration have negative effects on the prognosis, and isolated heterotrophic ossification seems to be related to a positive prognosis after surgical treatment.

Keywords - Elbow/injuries; Elbow/surgery; Wounds and injuries são: o alto grau de congruência, a complexidade de suas superfícies articulares e a grande sensibilidade tecidual ao trauma, especialmente da cápsula articular. Além da relação direta da rigidez articular do cotovelo com o trauma, má reabilitação e imobilização prolongada desnecessária, também, são fatores relacionados à perda de amplitude de movimento, e nos quais o ortopedista tem influência direta.

O envolvimento do paciente com o tratamento, também é citado como fator causal de rigidez articular do cotovelo, porém, para muitos autores essa não é uma causa de grande relevância.

Outras causas descritas incluem queimaduras e ossificação heterotópica, frequentemente consequente a traumatismos cranianos ${ }^{(3)}$.

1 - Diretor Geral do Instituto Nacional de Traumatologia e Ortopedia - (INTO), RJ.

2 - Médico do Centro de Cirurgia do Ombro e Cotovelo do Instituto Nacional de Traumatologia e Ortopedia - (INTO), RJ.

Trabalho realizado no Instituto Nacional de Traumatologia e Ortopedia - (INTO), RJ.

Correspondência: Marcus Vinícius Galvão, Av. Afrânio de Melo Franco, 141, sala 511, Leblon - 22430-060 - Rio de Janeiro, RJ. E-mail: mvgamaral@terra.com.br 


\section{Patologia}

Estudos experimentais em laboratório têm investigado as alterações bioquímicas e biológicas, que ocorrem nos tecidos periarticulares, como resposta ao trauma. Cohen et $a^{(4)}$ documentaram que cotovelos rígidos apresentam uma cápsula fina, com desorganização da matriz colágena, aumento de citocinas inflamatórias e infiltração fibroblástica, caracterizando uma condição fibrótica e inflamatória. Outros autores documentaram aumento na formação de colágeno do tipo cross-linked, associado à diminuição do conteúdo de proteoglicanos e água em articulações contraturadas, além de alterações na regulação do fator de crescimento beta 1 (TGF $\beta)$. Outro conceito importante que surge a partir das investigações mais recentes, sugere a existência de uma resposta individual a um trauma de mesma intensidade ${ }^{(5,6)}$.

\section{Quadro clínico}

A perda de amplitude de movimentos depende da natureza da lesão e seu tratamento. A perda da extensão é mais comum, porém, perda de flexão não é incomum, assim como, da rotação do antebraço ${ }^{(1)}$. A combinação da perda da extensão associada à perda da supinação do antebraço, representa limitação grave para determinadas atividades da vida diária ${ }^{(2)}$.

Em pacientes que apresentam luxações simples do cotovelo, a perda de movimento é puramente intrínseca, relacionada à contratura capsuloligamentar e ao dano muscular. Pacientes com fratura-luxação apresentam limitação dos movimentos do cotovelo, seja pela natureza da lesão ou pela reabilitação pós-operatória. Em alguns casos, a instabilidade articular, incongruência articular ou subluxação, principalmente úmero-ulnar, pode estar associada à limitação do arco de movimento ${ }^{(1,7)}$.

A dor não costuma ser um sintoma presente, exceto na presença de degeneração articular ou nos movimentos extremados. Por outro lado, dor em repouso pode sugerir infecção, principalmente em indivíduos já submetidos a cirurgias prévias ${ }^{(1)}$. Nesses casos, a dosagem da proteína $\mathrm{C}$ reativa e velocidade de hemossedimentação estará indicada.

Um dos parâmetros clínicos mais importantes para indicação de tratamento cirúrgico é a presença de sintomatologia relacionada ao nervo ulnar. Sinais de neuropatia indicam necessidade de neurólise e transposição anterior. Por vezes, a sintomatologia neuropática pode ser subclínica, devendo o examinador procurar identificar esses pacientes por meio de testes provocativos ${ }^{(1-3)}$.
O exame sob anestesia é controverso, quanto a suas indicações e eficácia. Morrey recomenda esse procedimento, em pacientes que durante o período pós-operatório não responderam à reabilitação, mesmo com uso de imobilizadores articulados, fisioterapia, e até aparelhos de mobilização articular contínua (Amac) ${ }^{(1)}$ (Figura 1).

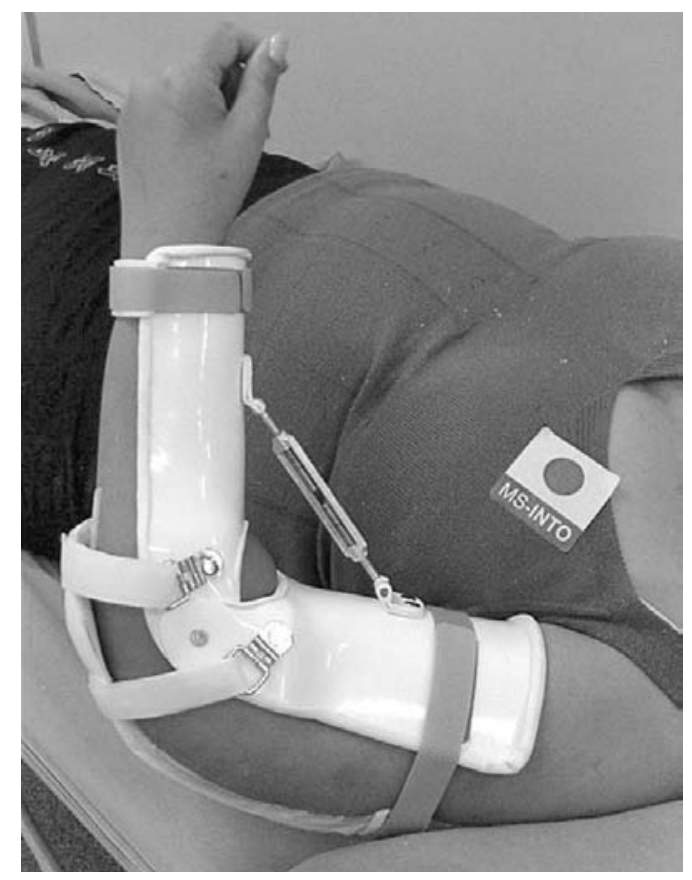

Figura 1 - Paciente utilizando imobilizador articulado com dispositivo para ganho passivo de amplitude de movimento (flexão)

\section{CLASSIFICAÇÃO}

Morrey $^{(1)}$ classificou a rigidez articular em dois grupos principais, baseando-se na etiologia e localização anatômica da contratura. Essa poderá ser extrínseca, quando se limita às partes moles ou a processos extraarticulares. As intrínsecas referem-se a processos articulares, como consolidações viciosas e doenças articulares degenerativas. Frequentemente, contraturas intrínsecas apresentam um componente extrínseco associado, sendo consideradas contraturas mistas ${ }^{(2)}$.

$\mathrm{Kay}^{(8)}$ descreveu outra classificação para a rigidez do cotovelo, baseada nos componentes envolvidos no processo. No tipo I existirá somente uma contratura isolada de partes moles. No II, contratura de partes moles associada à ossificação heterotópica. No tipo III, contratura de partes moles associada à fratura articular consolidada, sem desvio. No IV, a contratura de partes moles estará associada à consolidação viciosa de fratura articular e o tipo $\mathrm{V}$ refere-se à presença de barra óssea transarticular. 


\section{Exames complementares}

Na maioria dos casos, radiografias simples em anteroposterior e perfil do cotovelo são suficientes. Em contraturas maiores que $30^{\circ}$ a imagem em anteroposterior apresenta-se distorcida, nesses casos, as imagens oblíquas são opções adequadas. Além de deformidades articulares, deveremos avaliar o espaço articular, a qualidade da cartilagem articular, sua congruência, presença de ossificações heterotópicas e a localização de material de osteossíntese ${ }^{(3)}$.

Tomografia computadorizada deverá ser solicitada sempre que houver acometimento articular associado, que é mais grave quando acomete a articulação úmero-ulnar ${ }^{(2,3)}$ (Figura 2).

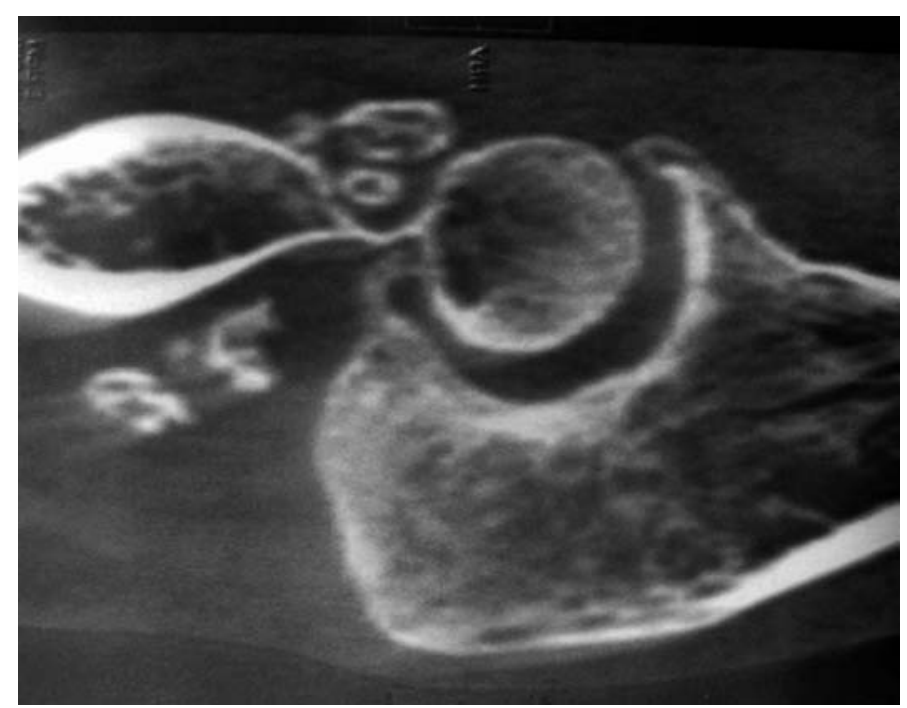

Figura 2 - Imagem em corte sagital de tomografia computadorizada do cotovelo demonstrando presença de corpos livres intra-articulares com degeneração articular ulnoumeral

Eletroneuromiografia é necessária quando existir suspeita clínica de neuropatia do nervo ulnar ${ }^{(3)}$.

Por outro lado, imagem de ressonância magnética é exame de exceção na propedêutica desta patologia ${ }^{(9)}$.

\section{Indicações cirúrgicas}

$\mathrm{O}$ arco funcional do cotovelo é definido como uma amplitude de movimento de flexo-extensão de $30^{\circ}$ a $130^{\circ}$ e pronossupinação de $50^{\circ}$ a $-50^{\circ(10)}$. Por outro lado, perda de $50 \%$ da mobilidade do cotovelo representa perda funcional de $80 \%$ da função da extremidade. Da mesma forma, contraturas em flexão maiores de $45^{\circ}$ determinam disfunção grave na capacidade de posicionar a mão no espaço.
Em resumo, a intervenção cirúrgica estará indicada em pacientes que apresentam uma amplitude de movimento no cotovelo, menor que $100^{\circ}$ de flexo-extensão ou de $50^{\circ}$ a $-50^{\circ}$ de pronossupinação. Porém, a indicação deve ser individualizada, de acordo com as necessidades funcionais de cada paciente e a habilidade do cirurgião.

Pacientes com rigidez articular do cotovelo, independente da etiologia, que apresentam sinais clínicos de neuropatia do nervo ulnar, devem ser tratados cirurgicamente com neurólise e transposição do nervo, associada à liberação articular do cotovelo. O comprometimento motor exige indicação cirúrgica absoluta ${ }^{(1,2)}$.

O grau de acometimento articular é o fator prognóstico mais importante, e a variável que orienta a modalidade terapêutica e resultados, que irá da liberação articular até a artroplastia de interposição ${ }^{(1)}$.

\section{TRATAMENTO}

A escolha da técnica a ser empregada depende da etiologia da rigidez articular e da experiência do cirurgião. Diversas modalidades de tratamento foram descritas na literatura especializada, porém com resultados inconsistentes.

\section{Tratamento incruento}

As modalidades não-operatórias, no manejo da rigidez pós-traumática do cotovelo, consistem no uso de imobilizadores articulados e fisioterapia. O tratamento conservador até o quarto mês não é de exceção, especificamente em quadros de rigidez não associadas a deformidades articulares e ossificação heterotópica. Apesar de alguns autores apresentarem ganhos de extensão de até $30^{\circ}$, com esse tipo de tratamento, esses resultados não foram reproduzidos pela maioria, sendo indicação de exceção. Manipulação sob anestesia em contraturas crônicas, além de não apresentar bons resultados, predispõe a formação de hematoma, dor, rigidez adicional e ossificação heterotópica ${ }^{(10,11)}$.

\section{Tratamento cruento}

O tratamento cirúrgico do cotovelo rígido estável pode ser realizado por via cruenta ou por técnica vídeoartroscópica. A opção por via cruenta é preferencial em casos em que exista uma retração acentuada de partes moles, onde a cirurgia artroscópica é tecnicamente difícil, e quando há indicação de ressecção de ossificação heterotópica, reconstrução articular ou artroplastia 
por interposição. Na presença de deformidade articular pós-traumática, a realização de osteotomias corretivas, associada à artrólise estará indicada. Artroplastia total do cotovelo é uma opção em indivíduos idosos, com baixa demanda, com limitação funcional e degeneração $\operatorname{articular}^{(1)}$.

A literatura ortopédica atual registra resultados funcionais semelhantes, nos grupos submetidos à liberação cruenta ou artroscópica, mas não existem trabalhos comparativos entre as técnicas, provavelmente pela dificuldade em identificar grupos homogêneos que possibilitem esse tipo de avaliação ${ }^{(12-14)}$.

A maioria das séries, não recomenda a realização de artrólise nas crianças e adolescentes, seja aberta ou artroscópica, em virtude da qualidade dos resultados obtidos nesse grupo de pacientes ${ }^{(15)}$. Porém, séries mais recentes afirmam que em pacientes sem incongruência ou degeneração articular os resultados são semelhantes aos da população adultaa ${ }^{(16)}$.

Dessa forma, independente da técnica, para que o tratamento cirúrgico seja eficiente é necessário identificar corretamente a causa da rigidez, o que possibilitará um planejamento cirúrgico específico e sequencial.

\section{Procedimento cruento}

Os cuidados no trato das partes moles periarticulares devem ser a principal preocupação quando utilizamos a técnica cruenta, principalmente em pacientes submetidos previamente à cirurgia, em virtude do risco de complicações pós-operatórias.

O percentual de complicações nas liberações cruentas é de aproximadamente 10 a 30\%, dependendo da natureza das alterações existentes e do tratamento empregado. As complicações descritas, em ordem de frequência, incluem: complicações de pele, infecção, neuropatia do nervo ulnar, ossificação heterotópica e dor ${ }^{(1,2,12)}$.

A seguir serão discutidas as diversas opções de acessos cirúrgicos utilizados para o tratamento da rigidez pós-traumática do cotovelo.

\section{Acesso anterior}

Urbaniak et al $^{(17)}$ popularizaram o acesso anterior ao cotovelo, principalmente para o tratamento da perda de extensão do cotovelo. Propõem, apenas, uma capsulotomia anterior transversa, em pacientes com contratura em flexão isolada. Outros autores realizam, por esse acesso, uma capsulectomia anterior associada a tenotomia do braquial. A grande limitação desse acesso é que ele não atua na limitação da flexão, e requer a identificação e proteção das estruturas neurovasculares. Apesar disso, essa via de acesso permite exposição direta da cápsula anterior e identificação de ossificações heterotópicas nessa topografia ${ }^{(1,17)}$.

\section{Acesso medial}

O acesso medial tem sua principal indicação, em pacientes com sinais de acometimento do nervo ulnar. Essa via de acesso, além de permitir o tratamento das alterações neurológicas do ulnar, também possibilita a abordagem de toda a cápsula anterior e o recesso posterior da articulação. Essa exposição é limitada e ineficiente, quando a doença acomete a articulação umerorradial e as estruturas laterais. Os reparos anatômicos desse acesso são o epicôndilo medial, o nervo ulnar e o septo intermuscular medial do braço. A chave para uma boa exposição é a separação do pronador redondo da massa flexora e sua elevação permitindo a visualização ampla da cápsula anterior. Para abordar a face posterior da articulação realiza-se liberação do nervo ulnar, o descolamento da porção medial do tríceps, permitindo-se a identificação dos osteófitos ${ }^{(1,2)}$.

\section{Acesso lateral limitado: o procedimento colunar}

O procedimento colunar descrito por Mansat e Morrey ${ }^{(18)}$ permite a abordagem anterior e posterior da articulação. A região anterior da articulação é identificada no intervalo entre as fibras distais do braquiorradial e do extensor radial longo do carpo. Dessa forma é possível ressecar os 2/3 laterais da cápsula anterior e incisar o seu 1/3 medial. Quando a cabeça radial estiver envolvida e existir indicação de sua ressecção, o acesso lateral estará indicado. Osteófitos do processo coronoide e da região anterior da extremidade distal do úmero podem ser, então, ressecados. A seguir, por meio da elevação da porção lateral do tríceps expõe-se a cápsula posterior, osteófitos posteriores, tecido fibroso que preenche a fossa olecraneana e osso heterotópico permitindo a ressecção adequada.

A complicação mais comum dessa abordagem é parestesia no território do nervo ulnar, seja por acesso agressivo às estruturas mediais, pela colocação de afastadores nessa topografia ou ainda, pelo ganho de movimento pós-operatório, principalmente de flexão, que expõe o nervo à tensão aumentada, tornando uma neuropatia subclínica em sintomática ${ }^{(18,19)}$. 


\section{Acesso posterior extenso}

Essa via de acesso está indicada em casos graves, quando a exposição medial e lateral é necessária ou em casos em que a superfície articular está acometida ${ }^{(1)}$. Realiza-se um acesso posterior de pele, sendo o nervo ulnar a primeira estrutura a ser identificada e liberada de seu leito. Em seguida, é realizada a dissecção do retalho cutâneo lateral, elevando a massa extensora da cápsula anterior, identificando-se e protegendo o complexo ligamentar lateral. Em seguida, é feito o descolamento do retalho medial e o nervo ulnar, previamente identificado, deverá ser protegido. Nos casos, em que persistir a limitação da flexão, após a liberação lateral, a ressecção da banda posterior do ligamento colateral medial estará indicada, por este ser um restritor importante da flexão a partir de $110^{\circ}$. Por esse acesso, a ressecção da cápsula medial também poderá ser realizada por visão direta, assim como eventual ossificação heterotópica ${ }^{(20)}$ (Figura 3).

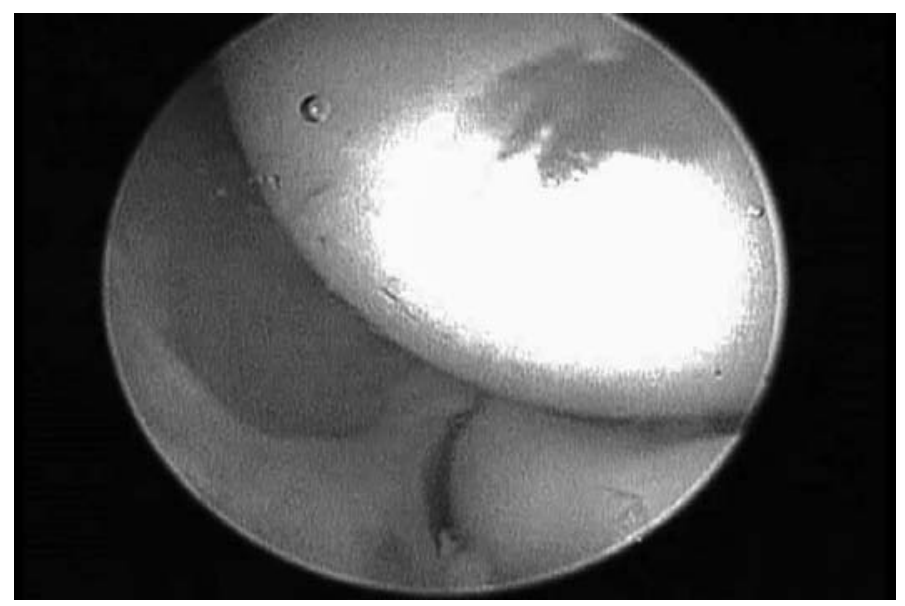

Figura 3 - Imagem de artroscopia do cotovelo visualizando o recesso posterolateral da articulação permitindo acesso à cabeça do rádio

\section{Procedimento artroscópico}

A cirurgia, por técnica vídeoartroscópica, para o tratamento do cotovelo rígido pós-traumático tem tornadose uma prática corrente, com resultados consistentes na literatura ortopédica. Porém, é um procedimento tecnicamente complexo para o cirurgião. Deve ser indicado, preferencialmente, nas contraturas menos graves, com perda menor que $15^{\circ}$ de extensão, e quando existirem corpos livres intra-articulares ${ }^{(13,14,21,22)}$.

O que torna o procedimento complexo é a proximidade das estruturas neurovasculares, com os tecidos periarticulares e os portais artroscópicos, associado à redução do volume capsular, que poderá ser de até $6 \mathrm{~cm}^{3}$, o que dificulta a realização da distensão hídrica da articulação, aumentando o risco de lesão das estruturas nobres ${ }^{(23)}$.

Nessa técnica, o passo inicial é a identificação e a ressecção de todos os corpos livres. A seguir, realiza-se a ressecção de osteófitos e ossos heterotópicos, preservando-se, integralmente, a estrutura capsular. Morrey ${ }^{(1)}$, preferencialmente, observa por meio do portal anteromedial e realiza a ressecção óssea através do portal anterolateral. O uso de retratores capsulares popularizados por Kelly et $a l^{(23)}$ é extremamente útil nesse momento. Após a realização do tempo ósseo descrito anteriormente, a cápsula anterior é liberada, de proximal para distal, sempre lateral ao processo coronoide, observando por meio do portal medial. Aspiração não deve ser utilizada, devendo-se usar somente fluxo gravitacional de saída. $\mathrm{O}$ nervo radial encontra-se em risco nesse momento, estando entre um a dois milímetros (mm) da cápsula articular. O risco de lesão nervosa é minimizado, quando realizamos a capsulectomia umeral, evitando-se trabalhar no tecido capsular que se encontra na topografia da cabeça do rádio. A seguir confeccionam-se o portal central posterior e o posterolateral, realizando-se a abordagem posterior na mesma sequência ${ }^{(21-23)}$.

Atualmente, diversas séries de casos têm apresentado resultados satisfatórios com índice de complicações equivalente às artrólises realizadas por técnica aberta $^{(13,14,23)}$ (Figura 4, A, B, C e D).

\section{Artroplastia de interposição}

A artroplastia de interposição, associada à artrólise deve ser considerada em pacientes jovens, que apresentam contratura mista com sinais radiográficos de degeneração das superfícies articulares de mais de 50\%, ou que necessitem de remodelagem das superfícies articulares por consolidação viciosa. Fáscia lata é o tecido mais comumente utilizado nesse procedimento, e deve ser suturado, cuidadosamente, ao redor de toda a superfície articular, comprometida por meio de sutura transóssea. A abordagem ao compartimento lateral do cotovelo, com liberação do complexo ligamentar lateral é possível com a exposição ampla da articulação, realizando-se um fulcro de abertura lateral sobre o ligamento colateral medial, que deverá estar íntegro. A ressecção da cabeça do rádio associada deve ser evitada, pois aumenta o risco de instabilidade pós-operatória. 

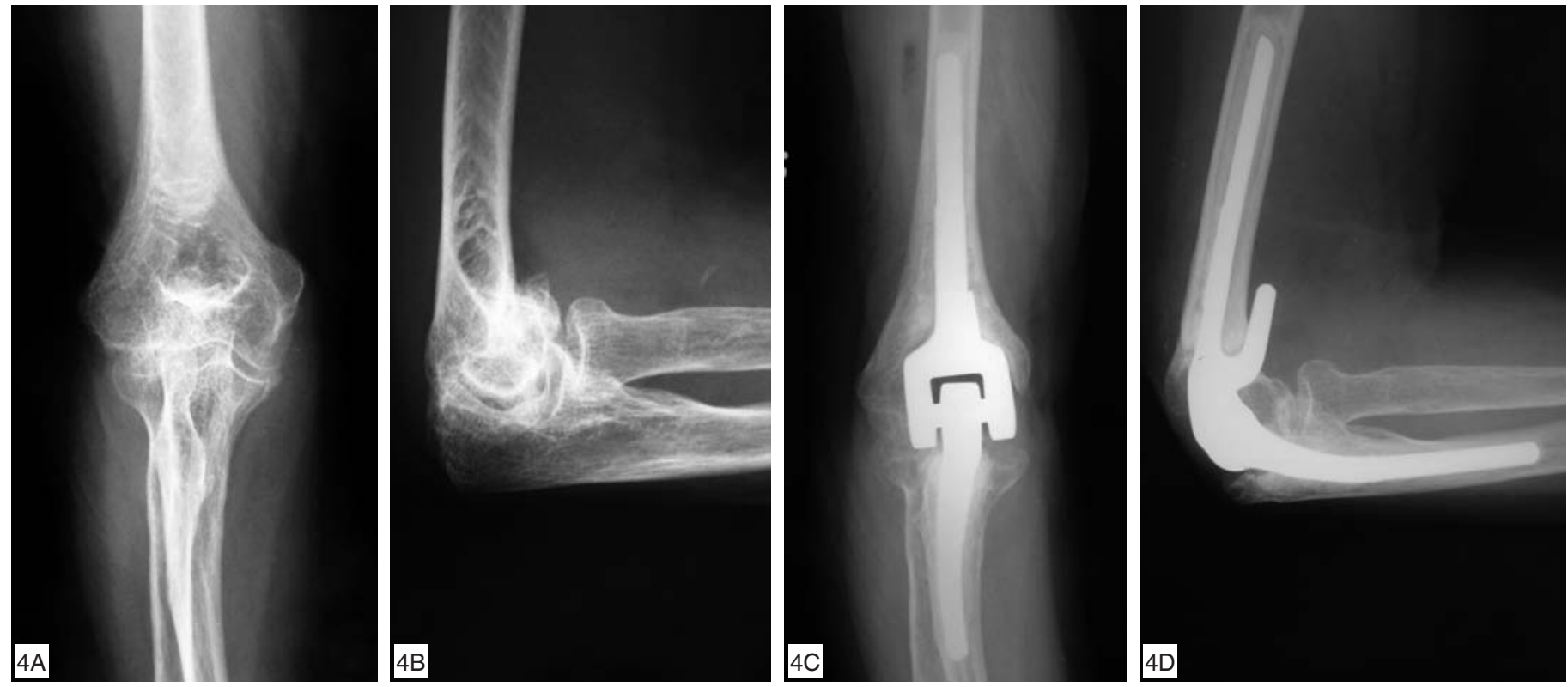

Figura 4 - Incidências radiográficas em anteroposterior $(A)$ e perfil $(B)$ de um paciente com anquilose articular submetido à artroplastia total semiconstrita do cotovelo (C) e (D)

O emprego do fixador externo articulado, por quatro a seis semanas é recomendado, propiciando distração articular, estabilidade e permitindo a mobilização precoce ${ }^{(20)}$.

\section{Artroplastia total de cotovelo}

Estará indicada em pacientes idosos, com mais de 65 anos, que apresentam restrição funcional grave do cotovelo, associada à degeneração das superfícies articulares.
Na literatura ortopédica há pouca informação a respeito desse procedimento. Mansat e Morrey ${ }^{(24)}$ relatam 76\% de resultados satisfatórios, porém, com 50\% de complicações, sendo dois casos de infecção profunda. Além disso, segundo Mansat e Morrey ${ }^{(24)}$ e Blaine et al $^{(25)}$, a artroplastia total do cotovelo, em pacientes que previamente foram submetidos a artroplastia de interposição, apresenta resultado e incidência de complicações comparadas às das séries de revisão de artroplastia total de cotovelo (Figura 5, A, B e C).
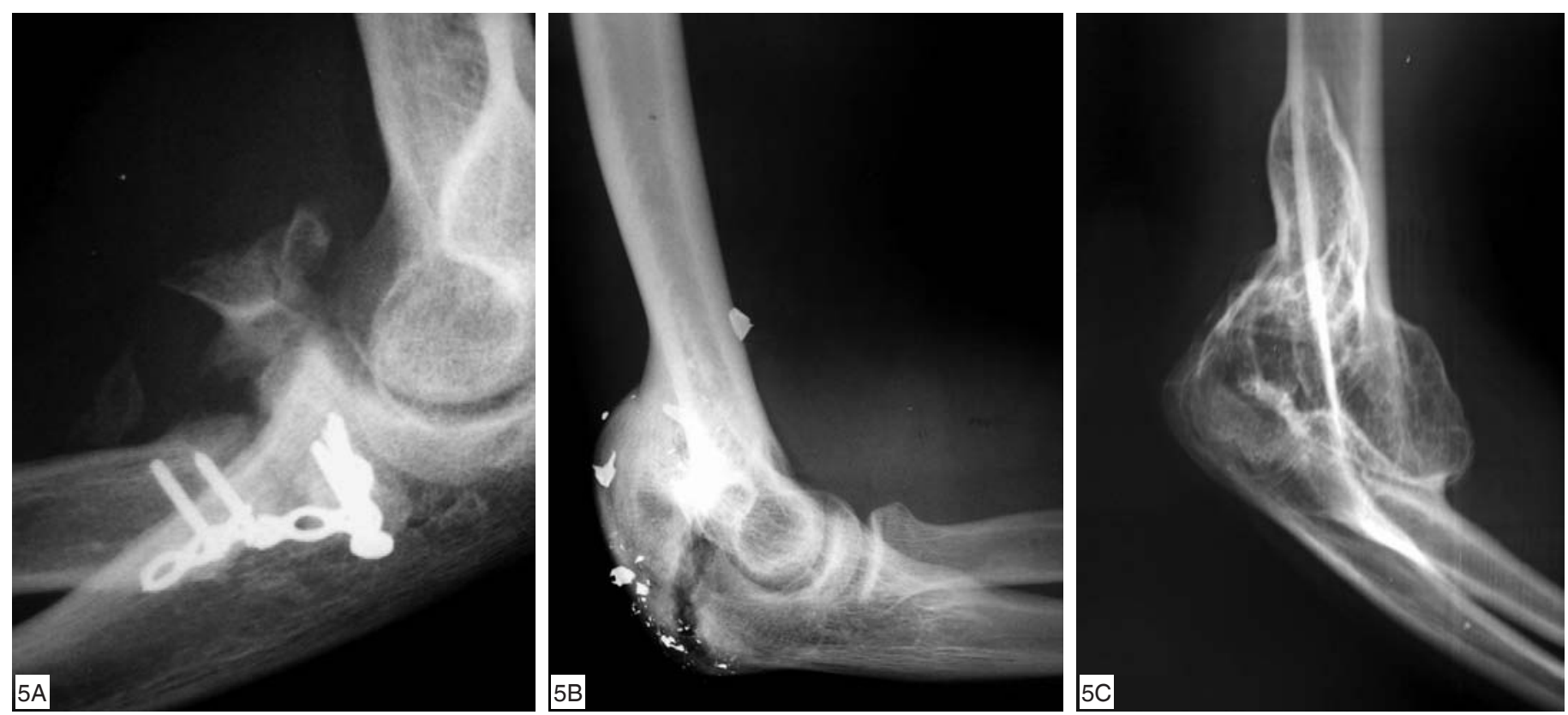

Figura 5 - Imagens radiográficas evidenciando diferentes tipos de ossificações heterotópicas de acordo com a classificação de Hasting. (A) Tipo I com ossificação heterotópica isolada em partes moles, (B) tipo IIA a presença de barra óssea incompleta e (C) tipo IIB anquilose articular 


\section{Ossificação heterotópica}

A ossificação heterotópica é um dos fatores relacionados à rigidez pós-traumática de cotovelo.

A eficácia da medicação inflamatória não hormonal e da radioterapia, na prevenção de ossificação heterotópica no cotovelo, não apresentam comprovação científica ${ }^{(26)}$.

Não existem, ainda, trabalhos controlados quanto ao uso da Indometacina combinado com os métodos contemporâneos de mobilização do cotovelo no pós-operatório. Acredita-se que, provavelmente, a utilização dos Amac e dos fixadores externos articulados, diminuiriam a incidência de ossificação heterotópica.

De toda forma, os cirurgiões que regularmente tratam o cotovelo rígido utilizam baixa dosagem de radiação, ou três a seis semanas de indometacina, 75mg diariamente, divididas em três tomadas.

Hastings e Graham ${ }^{(27)}$ propôs uma classificação da ossificação heterotópica, em três tipos relacionados com a extensão da formação de osso ectópico (Figura 6, A e B).

A maioria dos pacientes, que apresentam ossificação heterotópica são candidatos à intervenção cirúrgica. O momento ideal para a ressecção será definido pela presença de sinais radiográficos de maturidade da ossificação e tempo de evolução mínimo de quatro a seis meses ${ }^{(28,29)}$.

A utilização da cintilografia óssea tem valor limitado para avaliação da maturidade da ossificação, sendo pouco utilizada na prática clínica.

A ossificação heterotópica era considerada fator de mau prognóstico quando relacionada à rigidez articular do cotovelo ${ }^{(26)}$. Recentemente, alguns autores sugeriram haver evidência clínica, que a associação de rigidez do cotovelo com ossificação heterotópica apresentaria melhores resultados clínicos pós-operatórios ${ }^{(28,29)}$.

\section{Pós-operatório}

O tratamento pós-operatório do cotovelo rígido, depende da sua etiologia e do tipo de procedimento cirúrgico empregado.

Nos casos em que foi necessária a liberação do complexo ligamentar lateral ou nas artroplastias de interposição, o uso do fixador externo é útil, propiciando proteção a reconstruções ligamentares e articulares, permitindo a mobilização articular precoce, principalmente nas três semanas iniciais ${ }^{(1)}$.

Outra opção é a utilização do Amac, pouco disponível em nosso meio, mas, que permite, mobilização passiva da articulação com bons resultados, sempre de forma associada ao bloqueio contínuo do plexo braquial nos primeiros dias, propiciando uma articulação indolor que poderá ser mobilizada adequadamente ${ }^{(1,3,25)}$.

Uma forma de reabilitação mais acessível é o uso de imobilizadores articulados. Poderão ser utilizados com mobilização contínua ou intermitente, sendo esta última forma, reservada para casos em que se deseja privilegiar um movimento específico, seja extensão ou flexão ${ }^{(30)}$.

O valor da fisioterapia é questionável e controverso na literatura ortopédica, pois a potencial agressão a articulação, durante as sessões, causa dor e aumento do processo inflamatório, impedindo a recuperação da amplitude dos movimentos. Ela deve ser reservada para quando existir uma interação efetiva do cirurgião com o terapeuta, de forma que o mesmo esteja informado quanto ao procedimento realizado, os resultados e as limitações esperadas ${ }^{(1)}$.

Cabe ressaltar, que cada paciente necessita uma abordagem individualizada utilizando-se uma ou mais, das técnicas descritas.
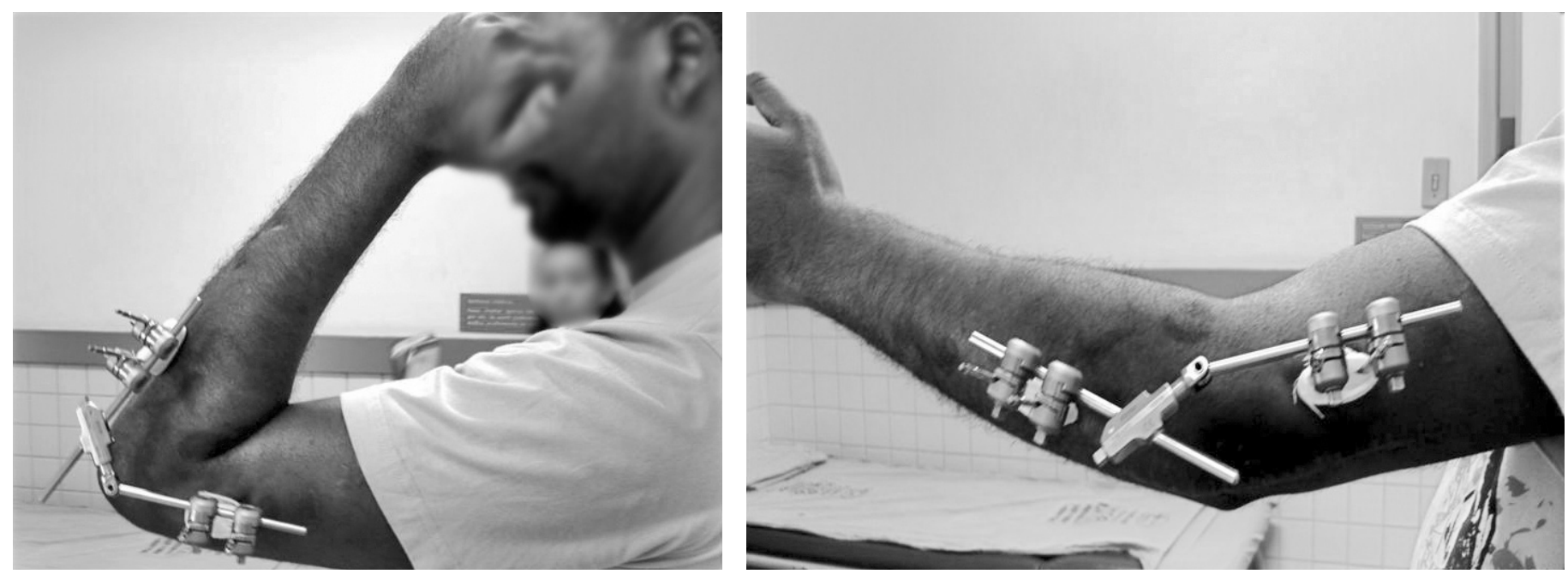

Figura 6 - Pós-operatório de artrólise aberta extensa mobilizando precocemente o cotovelo em regime ambulatorial utilizando um fixador externo articulado (A) e (B) 


\section{CONCLUSÃO}

Nos últimos 20 anos, dramáticas mudanças na abordagem da rigidez pós-traumática do cotovelo têm sido documentadas. O melhor entendimento das alterações patológicas e biomecânica articular permitiu o desenvolvimento de técnicas cirúrgicas mais adequadas. Mas os resultados pós-operatórios dependem da extensão da doença, tratamento empregado e experiência do cirurgião. Acreditamos que um melhor diagnóstico e tratamento das lesões agudas traumáticas do cotovelo, ainda são a melhor forma de prevenir este tipo de complicação.

\section{REFERÊNCIAS}

1. Morrey BF. The posttraumatic stiff elbow. Clin Orthop Relat Res. 2005; (431):26-35.

2. Jupiter JB, O'Driscoll SW, Cohen MS. The assessment and management of the stiff elbow. Instr Course Lect. 2003;52:93-111.

3. King GJ, Faber KJ. Posttraumatic elbow stiffness. Orthop Clin North Am. 2000;31(1):129-43.

4. Cohen MS, Schimmel DR, Masuda K, Hastings H 2nd, Muehleman C. Structural and biochemical evaluation of the elbow capsule after trauma. J Shoulder Elbow Surg. 2007;16(4):484-90

5. Akai M, Shirasaki Y, Tateishi T. Viscoelastic properties of stiff joints: a new approach in analyzing joint contracture. Biomed Mater Eng. 1993;3(2):67-73.

6. Younai S, Venters G, Vu S, Nichter L, Nimni ME, Tuan TL. Role of growth factors in scar contraction: an in vitro analysis. Ann Plast Surg. 1996;36(5):495-501.

7. Broberg MA, Morrey BF. Results of treatment of fracture-dislocations of the elbow. Clin Orthop Relat Res. 1987;(216):109-19.

8. Kay NR. Arthrolysis of the posttraumatic stiff elbow. In: Stanley D, Kay NR, editors. Surgery of the elbow: practical and scientific aspects. London: Edward Arnold; 1998. p. 228-34.

9. Libicher M, Freyschmidt J. [Radiological diagnosis in contracted elbow joint. Value of CT and MRI]. Orthopade. 2001;30(9):593-601.

10. Bonutti PM, Windau JE, Ables BA, Miller BG. Static progressive stretch to reestablish elbow range of motion. Clin Orthop Relat Res. 1994;(303):128-34.

11. Duke JB, Tessler RH, Dell PC. Manipulation of the stiff elbow with patient under anesthesia. J Hand Surg Am. 1991;16(1):19-24.

12. Marti RK, Kerkhoffs GM, Maas M, Blankevoort L. Progressive surgical release of a posttraumatic stiff elbow. Technique and outcome after 2-18 years in 46 patients. Acta Orthop Scand. 2002;73(2):144-50.

13. Ball CM, Meunier M, Galatz LM, Calfee R, Yamaguchi K. Arthroscopic treatment of post-traumatic elbow contracture. J Shoulder Elbow Surg. 2002;11(6):624-9.

14. Cohen AP, Redden JF, Stanley D. Treatment of osteoarthritis of the elbow: a comparison of open and arthroscopic debridement. Arthroscopy. 2000;16(7):701-6.

15. Stans AA, Maritz NG, O'Driscoll SW, Morrey BF. Operative treatment of elbow contracture in patients twenty-one years of age or younger. J Bone Joint Surg Am.2002;84(3):382-7.

16. Darlis NA, Kaufmann RW, Sotereanos DG. Open surgical treatment of post- traumatic elbow contractures in adolescent patients. J Shoulder Elbow Surg. 2006;15(6):709-15.

17. Urbaniak JR, Hansen PE, Beissinger SF, Aitken MS. Correction of post-traumatic flexion contracture of the elbow by anterior capsulotomy. J Bone Joint Surg Am. 1985;67(8):1160-4.

18. Mansat $P$, Morrey BF. The column procedure: a limited lateral approach for extrinsic contracture of the elbow. J Bone Joint Surg Am. 1998;80(11):1603-15.

19. Cohen MS, Hastings $\mathrm{H}$ 2nd. Post-traumatic contracture of the elbow. Operative release using a lateral collateral ligament sparing approach. J Bone Joint Surg Br. 1998;80(5):805-12.

20. Morrey BF. Post-traumatic contracture of the elbow. Operative treatment,including distraction arthroplasty. J Bone Joint Surg Am. 1990;72(4):601-18.

21. Savoie FH 3rd, Field LD. Arthroscopic management of the stiff elbow. In: Morrey $B F$, editor. The elbow and its disorders. 4th ed. Philadelphia: Saunders; 2008. p. 596-608.

22. Steinmann SP, King GJ, Savoie FH 3rd; American Academy of Orthopaedic Surgeons. Arthroscopic treatment of the arthritic elbow. J Bone Joint Surg Am.2005;87(9):2114-21.

23. Kelly EW, Morrey BF, O'Driscoll SW. Complications of elbow arthroscopy. J Bone Joint Surg Am. 2001;83(1):25-34.

24. Mansat $P$, Morrey BF. Semiconstrained total elbow arthroplasty for ankylosed and stiff elbows. J Bone Joint Surg Am. 2000;82(9):1260-8.

25. Blaine TA, Adams R, Morrey BF. Total elbow arthroplasty after interposition arthroplasty for elbow arthritis. J Bone Joint Surg Am. 2005;87(2):286-92.

26. Thompson HC 3rd, Garcia A. Myositis ossificans: aftermath of elbow injuries. Clin Orthop Relat Res. 1967;50:129-34.

27. Hastings $H$ 2nd, Graham TJ. The classification and treatment of heterotopic ossification about the elbow and forearm. Hand Clin. 1994;10(3):417-37.

28. Ring D, Jupiter JB. Operative release of ankylosis of the elbow due to heterotopic ossification. Surgical technique. J Bone Joint Surg Am. 2004;86 (Suppl 1):2-10.

29. Lindenhovius AL, Linzel DS, Doornberg JN, Ring DC, Jupiter JB. Comparison of elbow contracture release in elbows with and without heterotopic ossification restricting motion. J Shoulder Elbow Surg. 2007;16(5):621-5.

30. Bain GI, Mehta JA, Heptinstall RJ. The dynamic elbow suspension splint. J Shoulder Elbow Surg. 1998;7(4):419-21. 